\title{
Ichthyofauna of the lower course of the Negro river drainage, Patagonia Argentina
}

\author{
Mariano Soricetti ${ }^{1}$, Santiago Morawicki ${ }^{1,3}$, Fredy José Guardiola Rivas ${ }^{1,3}$, Catalina Guidi ${ }^{1,3}$, \\ Facundo Quezada ${ }^{1}$, Adriana Edith Almirón ${ }^{2}$, Patricio José Solimano ${ }^{1}$
}

1 Universidad Nacional de Río Negro, Centro de Investigaciones y Transferencia de Río Negro, Rotonda Cooperación y Ruta Provincial Nº 1, C.P 8500, Viedma, Río Negro, Argentina. 2 Universidad Nacional de La Plata, Facultad de Ciencias Naturales y Museo, División Zoología Vertebrados, Paseo del Bosque s/n, C.P 1900, La Plata, Buenos Aires, Argentina. 3 Consejo Nacional de Investigaciones Científicas y Técnicas (CONICET), Centro de Investigaciones y Transferencia de Río Negro, Rotonda Cooperación y Ruta Provincial N 1 , C.P 8500, Viedma, Río Negro, Argentina. Corresponding author: Mariano Soricetti, msoricetti@unrn.edu.ar

\begin{abstract}
The drainage of the Negro River is the most important watercourse in the Patagonia ecoregion and, together with the Colorado river basin, define an ecotone, i.e., a zoogeographic transition, where coexist the Brazilian and Patagonian lineages of freshwater fishes. The Patagonia ecoregion has 29 fish species, 15 native and the remaining introduced. For this study, the ichthyofauna of seven locations in the lower course of the Negro river drainage were sampled along two years. Gillnets, coastal trawls, cast nets, river trammel nets and fishing rods were used to catch specimens. A total of 13 species belonging to nine orders and 11 families were collected. The families Atherinopsidae and Characidae show the highest species richness and one exotic species, Cyprinus carpio Linnaeus, 1758, was recorded. The origin and distribution of some species is discussed, considering the role of human action and certain environmental factors.
\end{abstract}

\section{Keywords}

Biodiversity, ichthyogeographic areas, introduced species, inventory, native species.

\section{Introduction}

South America has the richest freshwater fish fauna in the world, with more than 5,100 species which represent about one third of all freshwater fish worldwide (Reis et al. 2016). South America belongs to the Neotropical region and its freshwater fauna is distributed in 51 ecoregions defined by hydrographic limits (Abell et al. 2008; Albert and Reis 2011). These ecoregions in turn form 13 basin complexes grouped by hydrologic and biogeographic units (Reis et al. 2016). In the Neotropics there is a north-south gradient of species richness that presents its highest values in the Amazon-OrinocoGuyana core, and decreases as latitude increases (Albert and Reis 2011). In addition, it should be noted that as observed in Albert and Reis (2011), there are ecoregions with high percentages of endemism, such as the Maracaibo, Titicaca, and Patagonia. The Negro river drainage is in the north of Patagonia ecoregion, within Southern Cone basin complex, and is the most important watercourse in this ecoregion (Fig. 1).

The fish fauna of the Patagonia ecoregion is quite 


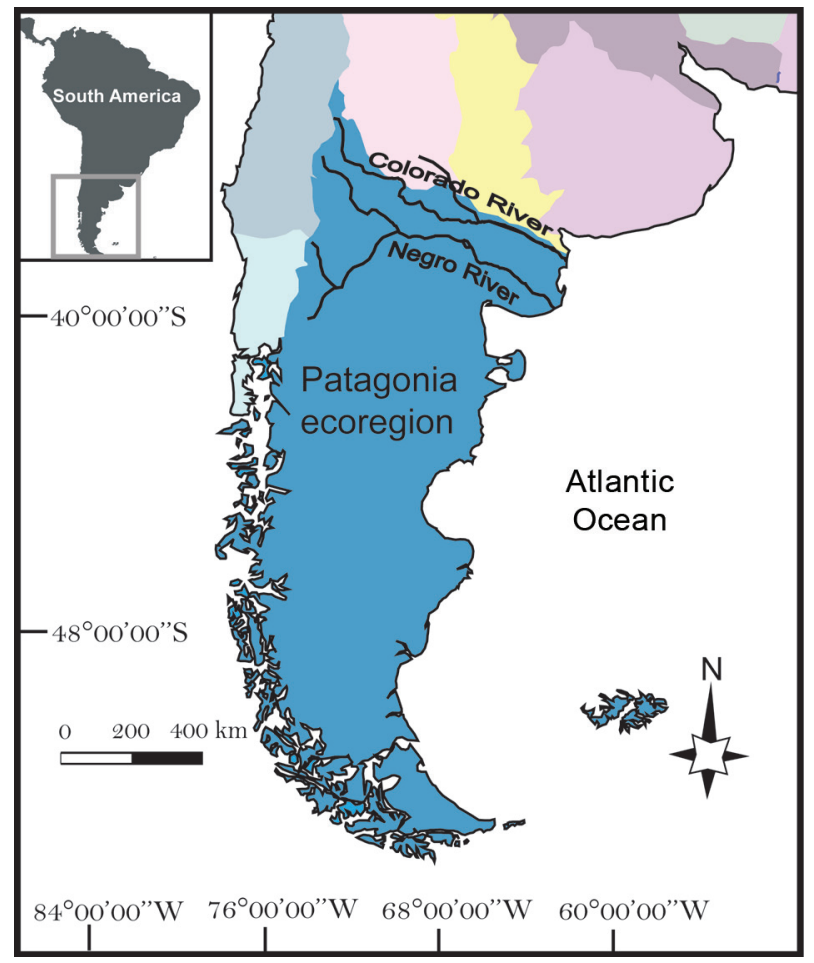

Figure 1. Location of the Negro river drainage within the Patagonia ecoregion. Modified from Abell et al. (2008).

distinct from that of the rest of the Neotropical region in terms of diversity of families and subfamilies (Malabarba and Malabarba 2008; Cussac et al. 2009). Climatic conditions, recent origin, altitude, and irregular flow of rivers contribute to the pauper freshwater fauna of Patagonia ecoregion and its dissimilarity to other parts of South America (Géry 1969). In other biogeographical frameworks, this led to the proposal of dividing the Neotropics into two subregions, the Brazilian subregion and the Austral subregion, where the Patagonian ichthyogeographic province was located (Ringuelet 1975). The difference between the Patagonia ecoregion and the rest of the ecoregions related to the great basins of the Neotropics is very large, even at the family and genus levels.

Species from Brazilian and Patagonian lineages coexist along the basins of the Colorado and Negro rivers, defining a zone of zoogeographic transition recognized as an ecotone (Arratia et al. 1983; Almirón et al. 1997). The specific richness of the Negro and Colorado river basins is higher than the other Patagonian rivers as a consequence of the historical connections of these systems with northern aquatic systems that resulted in the presence of Characiformes and Cyprinodontiformes in these rivers (Cione and Barla 1997; Casciotta et al. 1999; Bruno et al. 2013). Questions arise regarding the number of species present in these basins, the extent of their distribution areas, and the cause of their presence in the rivers, whether natural or introduced by anthropogenic activities (Baigún et al. 2002).

Patagonian fauna and landscapes have been modelled by natural and anthropogenic processes, such as the Gondwanic inheritance, the elevation of the Andes, the
Pleistocene ice, volcanic activity, introduction of exotic fauna, and climate change (Pascual et al. 2007). The Patagonia ecoregion has 29 species recorded, of which only 15 are native (Baigún and Ferriz 2003), and 14 introduced. In the latter case, species came from North America, Europe, or were translocated from other ecoregions of South America, and at least 11 of them have established self-sustaining populations (Pascual et al. 2007). Movement of species beyond their natural distribution range is one of the most ecologically damaging human activities (Lodge 1993; Wilcove et al. 1998; Koehn 2004). The introduction of exotic species could lead to a reduction in the diversity of the assemblage (Rahel 2000; Jackson 2002) because of alteration, modification, or interruption in the trophic chain (Vander Zanden et al. 1999; Zambrano et al. 2006). The introduction of fish species may be intentional-as the introduction of fish for recreational fishing - or incidental, such as the escape of individuals from fish farms or ballast water from boats.

There are few general studies on the ichthyofauna of Patagonia (Baigún and Ferriz 2003; Pascual et al. 2007; Aigo et al. 2008) and even fewer contributions on the ichthyofauna of the Negro river drainage (Alvear et al. 2007; Pérez and López Cazzorla 2008). This study provides information on fish diversity in the lower course of the Negro river drainage and updates data on the latitudinal distribution of some species.

\section{Methods}

Study area. The Negro River originates at the confluence of the Limay and Neuquén rivers, runs $700 \mathrm{~km}$ in a west-east direction, and ends in the Atlantic Ocean (Aparicio and Difrieri 1958; Alvear et al. 2007). It has an average flow of $845 \mathrm{~m}^{3} / \mathrm{s}$ (Pascual et al. 2007) and represents the most important watercourse in Patagonia region. The Negro River course crosses the Upper, Middle and Lower Valley of the homonymous province (Alvear et al. 2007). The Lower Valley extends along a strip that is $100 \mathrm{~km}$ long and approximately $8 \mathrm{~km}$ wide, from the "Primera Angostura" of the Negro river drainage, near the town of Guardia Mitre, to its mouth in the Atlantic Ocean (Berasategui 2002). The Inferior Valley Development Institute is a large irrigated area of more than 40,000 ha located upstream of Viedma city.

Data collection. Sampling of fish in the lower course of the Negro river drainage was carried out from January 2016 to December 2017. Seven locations were determined, each showing distinct characteristics of flow, and stream bed (Table 1; Fig. 2). Samples were obtained using a river trammel of $20 \mathrm{~m}$ long and $1 \mathrm{~m}$ high, with an external mesh of $300 \mathrm{~mm}$ and internal of $110 \mathrm{~mm}$; a 20 $\mathrm{m}$ coastal trawl with $5 \mathrm{~mm}$ mesh size; a cast net of $5 \mathrm{~m}$ diameter with $7 \mathrm{~mm}$ mesh; two gillnets of $25 \mathrm{~m}$ in length with $30 \mathrm{~mm}$ and $60 \mathrm{~mm}$ mesh sizes, and fishing rods. Samples were collected in tributaries of second and third 


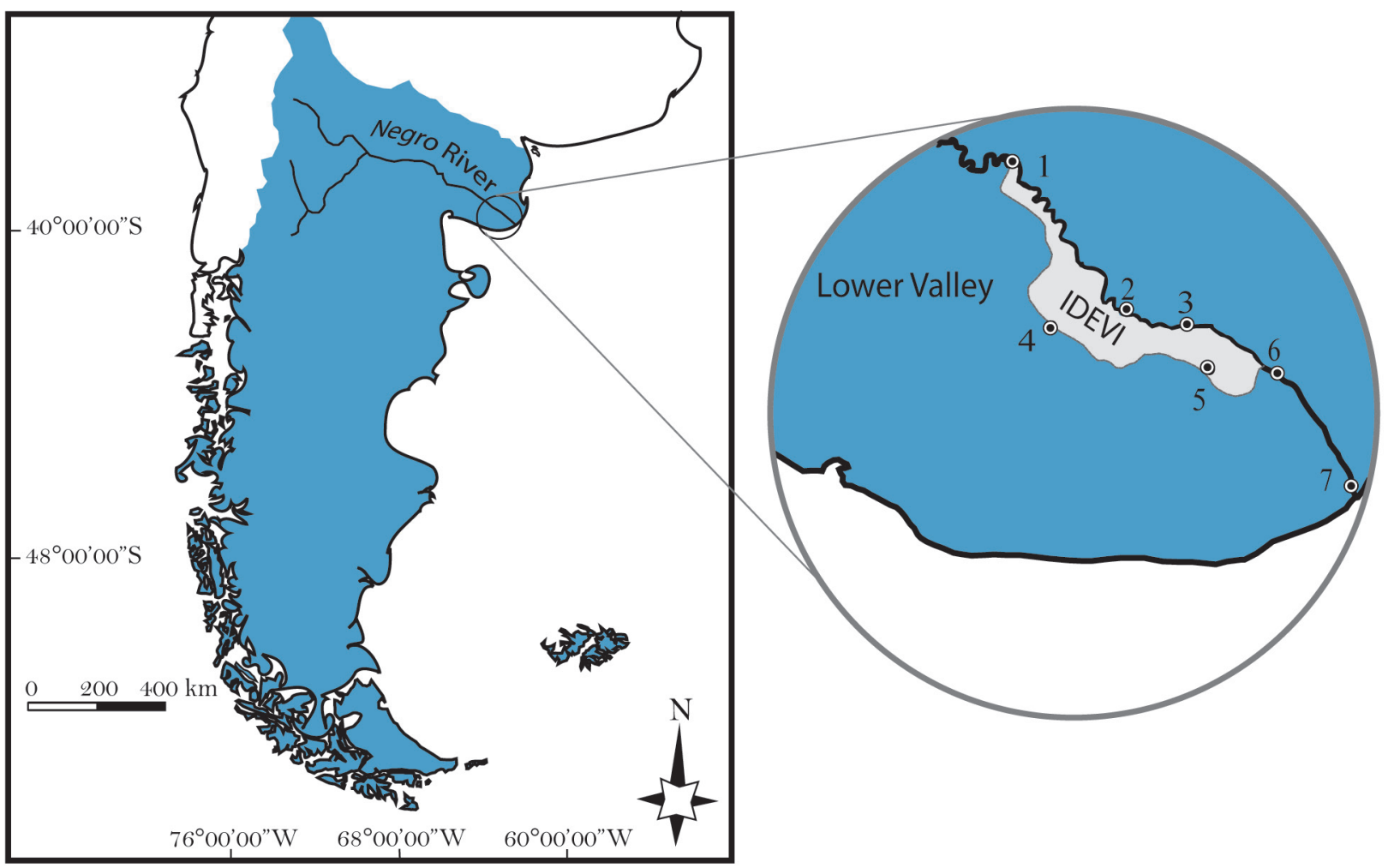

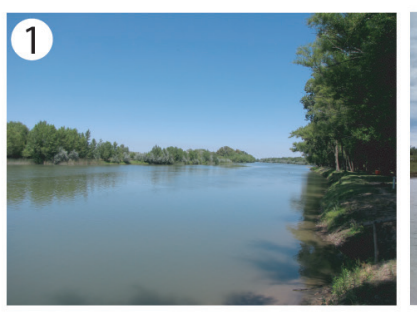

5

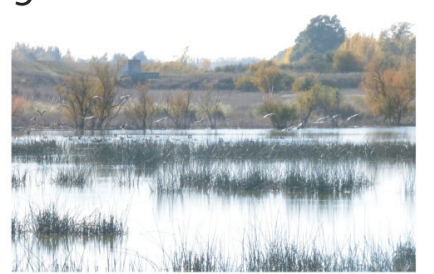

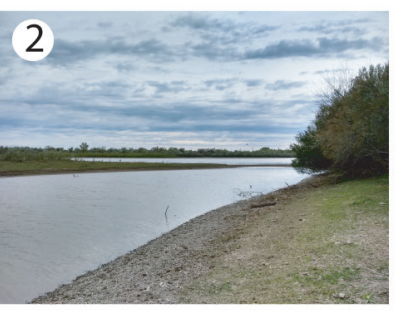

6

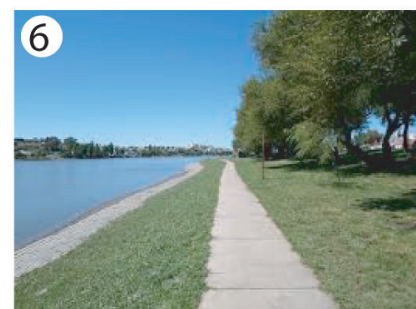

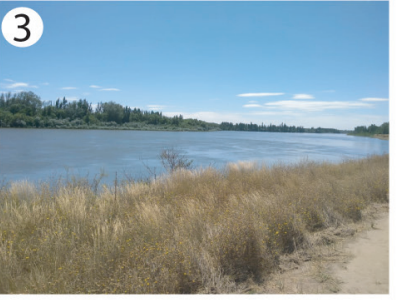
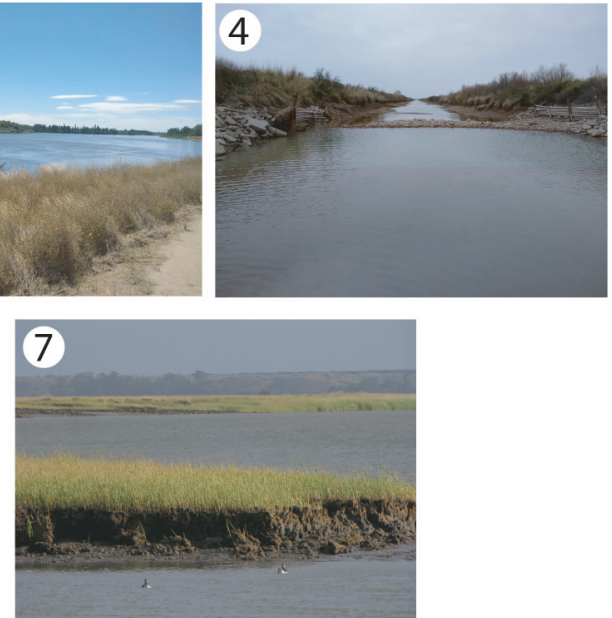

Figure 2. Sampling sites on the lower course of the Negro river drainage. Abbreviation: IDEVI, Inferior Valley Development Institute.

orders, coastal areas of the main course of the drainage, a small shallow lagoon, and irrigation and drainage channels.

The specimens were euthanized with an overdose of benzocaine, and their measurement of the total body length (TL) were taken later. All body measurements presented in material examined of the annotated species list are referred to the body total length. In the laboratory, specimens were fixed in $10 \%$ formaldehyde and preserved in $70 \%$ alcohol. Taxonomic identifications of the specimens were based on Dyer (2000), Casciotta et al. (2005), Díaz de Astarloa et al. (2006), Rosso (2006), Miquelarena et al. (2008), and Almirón et al. (2015). Collection permits were granted by the Ministry

Table 1. List of sampling sites on the lower course of the Negro river drainage and environmental features.

\begin{tabular}{lllll}
\hline Site & Latitude $(\mathbf{S})$ & Longitude $(\mathbf{W})$ & Flow conditions & Stream bed \\
\hline 1 & $40^{\circ} 26^{\prime} 18^{\prime \prime}$ & $063^{\circ} 40^{\prime} 54^{\prime \prime}$ & Alternating backwater and rapid water flow & Gravel and sand \\
2 & $40^{\circ} 42^{\prime} 48^{\prime \prime}$ & $063^{\circ} 23^{\prime} 08^{\prime \prime}$ & Alternating backwater, rapid water flow and pools & Gravel and sand \\
3 & $40^{\circ} 43^{\prime} 30^{\prime \prime}$ & $063^{\circ} 14^{\prime} 02^{\prime \prime}$ & Rapid water flow & Sandy \\
4 & $40^{\circ} 43^{\prime} 15^{\prime \prime}$ & $063^{\circ} 36^{\prime} 02^{\prime \prime}$ & Straight channel with low water flow & Clayey \\
5 & $40^{\circ} 49^{\prime} 26^{\prime \prime}$ & $063^{\circ} 09^{\prime} 51^{\prime \prime}$ & Pools & Clayey \\
6 & $40^{\circ} 48^{\prime} 10^{\prime \prime}$ & $062^{\circ} 59^{\prime} 36^{\prime \prime}$ & Rapid water flow & Sand and gravel \\
7 & $41^{\circ} 01^{\prime} 12^{\prime \prime}$ & $062^{\circ} 47^{\prime} 54^{\prime \prime}$ & Alternating rapid water flow and backwaters & Sandy, slightly steep banks \\
\hline
\end{tabular}


of Agriculture, Livestock and Fisheries of the Province of Río Negro (Resolution SSP No. 013/18), and by the Secretary of Environment and Sustainable Development (Resolution No. 127 of February 3, 2016) of the Province of Río Negro. All the specimens are deposited in the ichthyological collection of the Patagonian Museum of Natural Sciences 'Juan Carlos Salgado' (MPCN), General Roca, Province of Río Negro, Argentina.

\section{Results}

Thirteen species corresponding to nine orders and 11 families were recorded (Fig. 3, Table 2): Odontesthes bonariensis (Valenciennes, 1835), O. hatcheri (Eigenmann, 1909), Cheirodon interruptus (Jenyns, 1842), Psalidodon pampa (Casciotta, Almirón \& Azpelicueta, 2005), Cyprinus carpio Linnaeus, 1758, Jenynsia lineata (Jenyns, 1842), Cnesterodon decemmaculatus (Jenyns, 1842), Galaxias maculatus (Jenyns, 1842), Mugil liza (Valenciennes, 1863), Percichthys trucha (Cuvier \& Valenciennes, 1840), Paralichthys brasiliensis (Ranzani, 1840), Genidens barbus (Lacepède, 1803), and Corydoras paleatus (Jenyns, 1842).

The orders Atheriniformes, Characiformes, Cyprinodontiformes, and Siluriformes were represented by two species each. Among them, the families Atherinopsidae and Characidae were the richest, with two species each. The remaining families Anablepidae, Ariidae, Callichthyidae, Cyprinidae, Galaxiidae, Mugilidae, Paralichthyidae, Percichthyidae, and Poeciliidae were represented by only one species each. Of the total species captured, only three are freshwater species of the Patagonia ecoregion (23.1\%), six species are native from other Neotropical ecoregions (46.1\%), three species are originally marine with freshwater river intrusion (23.1\%), and one species is exotic (7.7\%) (Fig. 4).

The list of species and its meristic and morphometric characteristics used to identification are given below.

Order Atheriniformes

Family Atherinopsidae

\section{Odontesthes bonariensis (Valenciennes, 1835)} Figure 3A

Material examined. ARGENTINA • 1 o, $110 \mathrm{~mm}$; Site 4; 4043'15"S, 06336'02"W, 23 May 2017; P. Solimano leg.; MPCN-Ict 1. • 1 , 184 mm; Site 4; 4043'15"S, $063^{\circ}$ 36'02"W; 23 May 2017; P. Solimano leg.; MPCN-Ict 14. - 1 †, 162 mm; Site 4; 4043'15"S, 06336'02"W; 24 Jan. 2017; F. Guardiola Rivas leg.; MPCN-Ict 15. • 1 ð̄, 205 $\mathrm{mm}$; Site 6; 40 $48^{\prime} 10^{\prime \prime} \mathrm{S}, 062^{\circ} 59^{\prime} 36^{\prime \prime} \mathrm{W} ; 24$ Jan. 2017; B. Rodriguez leg.; MPCN-Ict 16. • 1 \%, $237 \mathrm{~mm}$; Site 6; 4048'10"S, 062 59'36"W; 23 May 2017; P. Solimano leg.; MPCN-Ict 17. • 1 ふ, 264 mm; Site 6; 4048'10"S, $062^{\circ} 59^{\prime} 36^{\prime \prime} \mathrm{W}$; 24 Jan. 2017; M. Soricetti leg.; MPCN-Ict 18 .

Identification. Body elongated. Body scales small (10-
12 dorsal scales) with smooth posterior edge. Mouth with a medial tooth patch on vomer; three rows of teeth in the oral jaws, the teeth of outer rows larger and more robust. Posterodorsal edge of the opercular bone straight. Thirty to 40 gill rakers on the lower branch of the first branchial arch. First dorsal fin origin coincident or slightly anterior to the vertical that passes through the anus; five or less interdorsals; last ray of the anal fin anterior to vertical passing through the last ray of the second dorsal fin. Three rows of subocular scales; scales present between the anterior rays of the anal and second dorsal fins.

\section{Odontesthes hatcheri (Eigenmann, 1909)}

Figure 3B

Material examined. ARGENTINA 1 ô, $98 \mathrm{~mm}$; Site 1; 40²6'18"S, 06340'54"W; 23 Nov. 2016; P. Solimano leg.; MPCN-Ict 2. • 1 , $136 \mathrm{~mm}$; Site 1; 40²6'18"S, 06340'54"W; 23 Nov. 2016; P. Solimano leg.; MPCN-

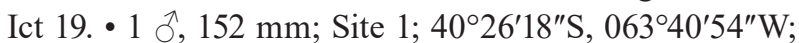
23 Nov. 2016; M. Soricetti leg.; MPCN-Ict 20. • 1 ふे, 212

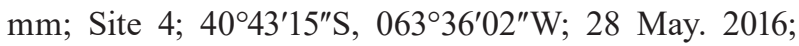
P. Solimano leg.; MPCN-Ict 21. • 1 +, 258 mm; Site 4; 4043'15"S, 063³6'02"W; 23 May. 2017; P. Solimano leg.; MPCN-Ict 22. • 1 q, 283 mm; Site 4; 4043'15"S, $063^{\circ} 36^{\prime} 02^{\prime \prime} \mathrm{W} ; 23$ May. 2017; M. Soricetti leg.; MPCN-Ict

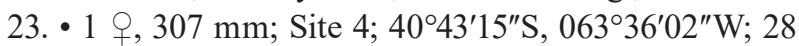
Jun. 2017; P. Solimano leg.; MPCN-Ict 24.

Identification. Body elongated. Adults with the upper jaw prognathous, premaxilla protractil anteroventrally and strong pharyngeal jaws with molariform teeth. Operculum with fenestrated inner face and convex posterodorsal edge. Dorsal edge of operculum continuing on the laminar ridge of dorsal operculum process. Three or more rows of teeth in the oral jaws; no teeth in vomer; 21-25 gill rakers on the lower branch. First dorsal fin coincident with the posterior end of the pelvic fins; six or more interdorsal bones in continuous series. Small body scales (15 dorsal scales) with smooth posterior border. Three rows of subocular scales; scales also present on the interoperculum and between the anterior rays of the anal fin, but absent in the second dorsal fin. Posterior margin of body scales lined with a row of melanophores giving a very dark appearance to the specimens.

Order Characiformes

Family Characidae

\section{Cheirodon interruptus (Jenyns, 1842)}

Figure 3C

Material examined. ARGENTINA 1 o, $64 \mathrm{~mm}$; Site 2; $40^{\circ} 42^{\prime} 48^{\prime \prime} \mathrm{S}, 063^{\circ} 23^{\prime} 08^{\prime \prime} \mathrm{W}$; 15 Oct. 2016; F. Guardiola Rivas leg.; MPCN-Ict 3. 1 ㅇ, 39 mm; Site 2; $40^{\circ}$ $42^{\prime} 48^{\prime \prime} \mathrm{S}, 063^{\circ} 23^{\prime} 08^{\prime \prime} \mathrm{W}$; 22 Nov. 2016; F. Guardiola Rivas leg.; MPCN-Ict 25. 1 + , 42 mm; Site 2; 4042'48"S, $063^{\circ} 23^{\prime} 08^{\prime \prime} \mathrm{W}$; 22 Nov. 2016; F. Guardiola Rivas leg.; MPCN-Ict 26. • 1 ô, $45 \mathrm{~mm}$; Site 2; 4042'48"S, $063^{\circ}$ 23'08"W; 13 Dec. 2016; M. Soricetti leg.; MPCN-Ict 27. 1 đo, $51 \mathrm{~mm}$; Site $4 ; 40^{\circ} 43^{\prime} 15^{\prime \prime} \mathrm{S}, 063^{\circ} 36^{\prime} 02^{\prime \prime} \mathrm{W} ; 21$ 


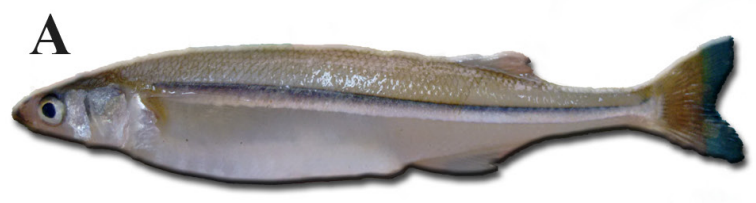

B

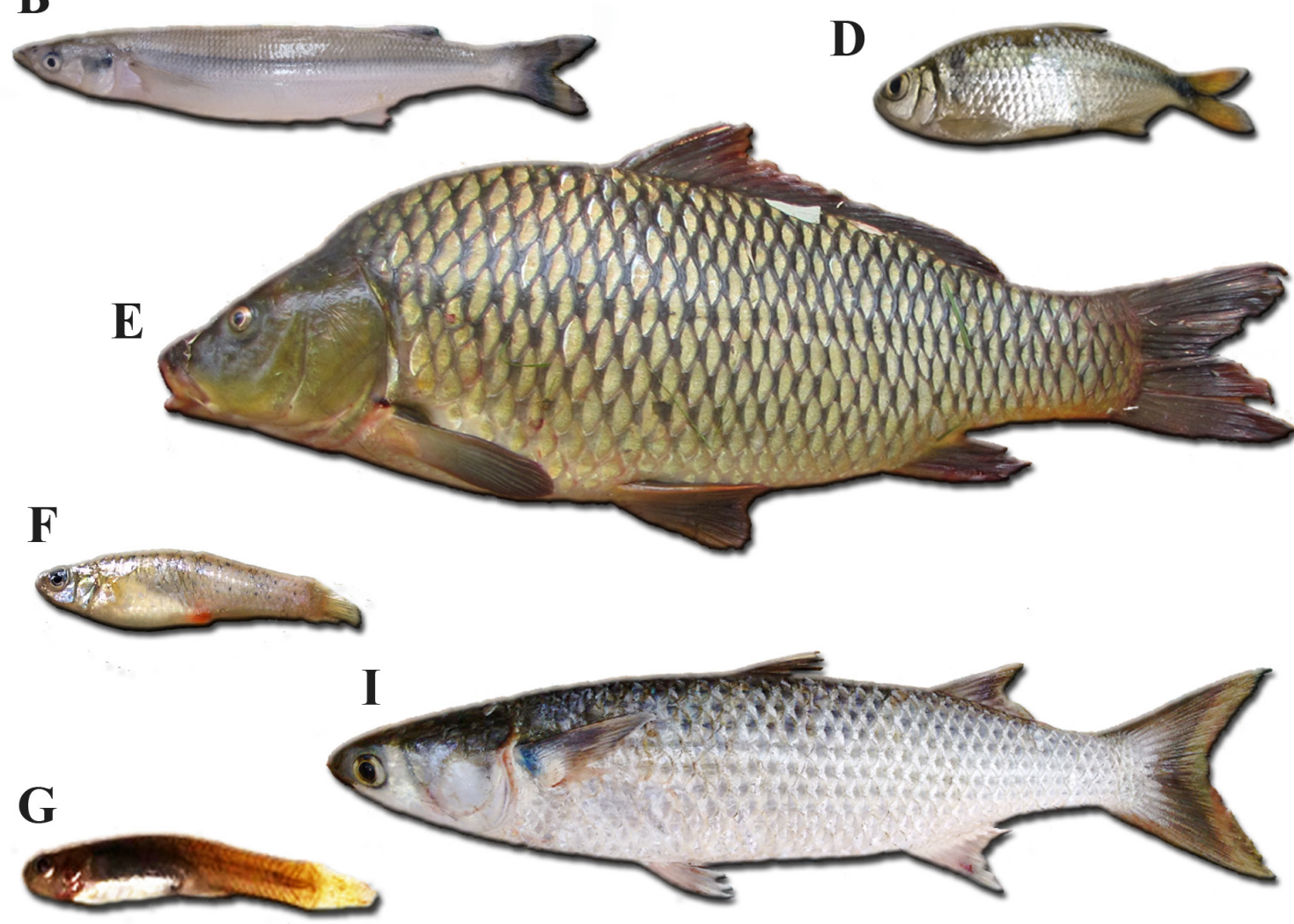

\section{H}

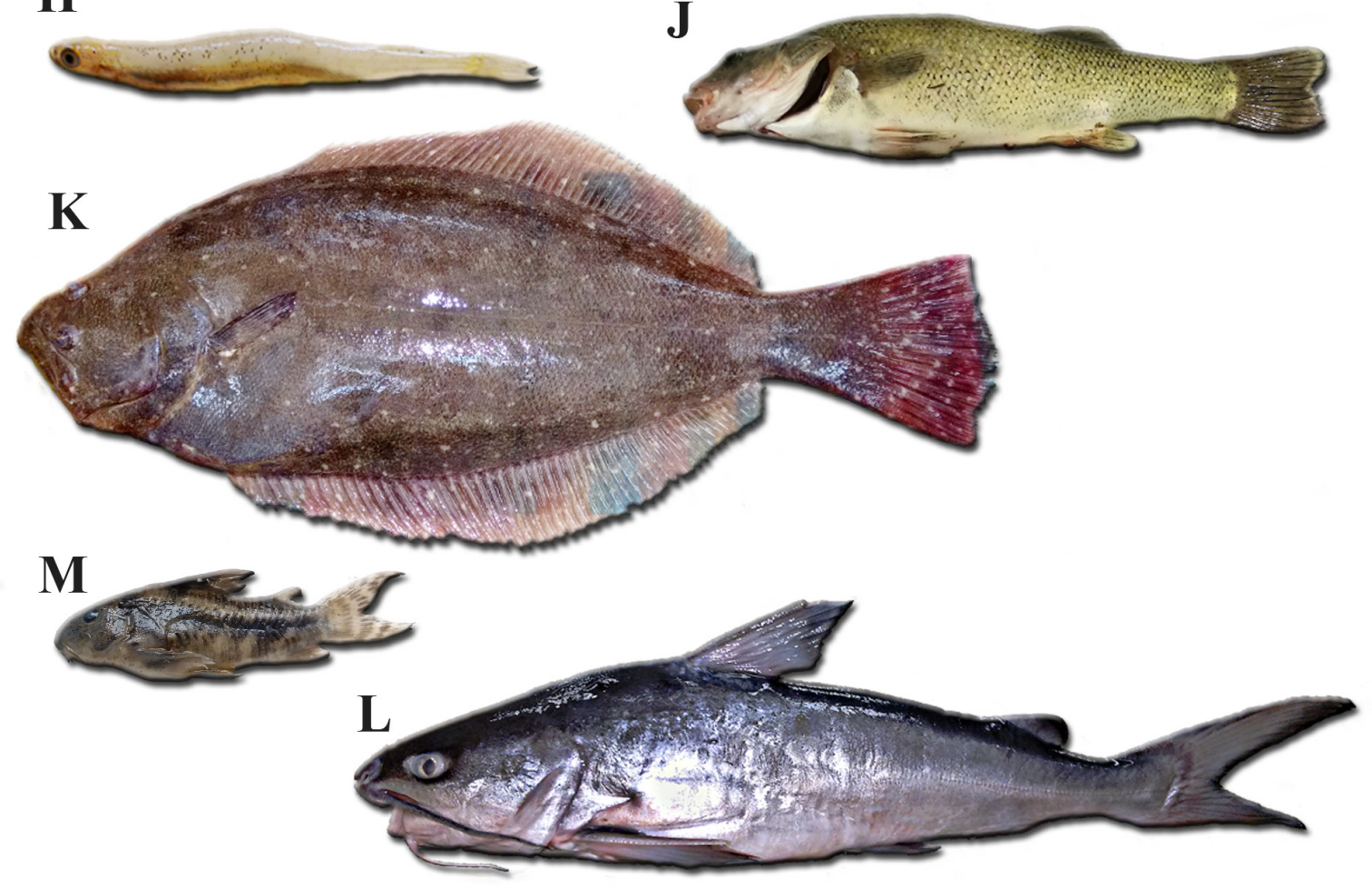

Figure 3. Fishes of the lower course of the Negro river drainage. A. Odontesthes bonariensis, MPCN-Ict 1, $110 \mathrm{~mm}$ TL. B. O. hatcheri, MPCNIct 2, $98 \mathrm{~mm}$ TL. C. Cheirodon interruptus, MPCN-Ict 3, $64 \mathrm{~mm}$ TL. D. Psalidodon pampa, MPCN-Ict 4, $73 \mathrm{~mm}$ TL. E. Cyprinus carpio, MPCN-Ict 5, $418 \mathrm{~mm}$ TL. F. Jenynsia lineata, MPCN-Ict 6, $59 \mathrm{~mm}$ TL. G. Cnesterodon decemmaculatus, MPCN-Ict 7, $36 \mathrm{~mm}$ TL. H. Galaxias maculatus, MPCN-Ict 9, $57 \mathrm{~mm}$ TL. I. Mugil liza, MPCN-Ict 8, $501 \mathrm{~mm}$ TL. J. Percichthys trucha, MPCN-Ict 10, $272 \mathrm{~mm}$ TL. K. Paralichthys brasiliensis, MPCN-Ict 11, 385 mm TL. L. Genidens barbus, MPCN-Ict 13, 320 mm TL. M. Corydoras paleatus, MPCN-Ict 12, 75 mm TL. 


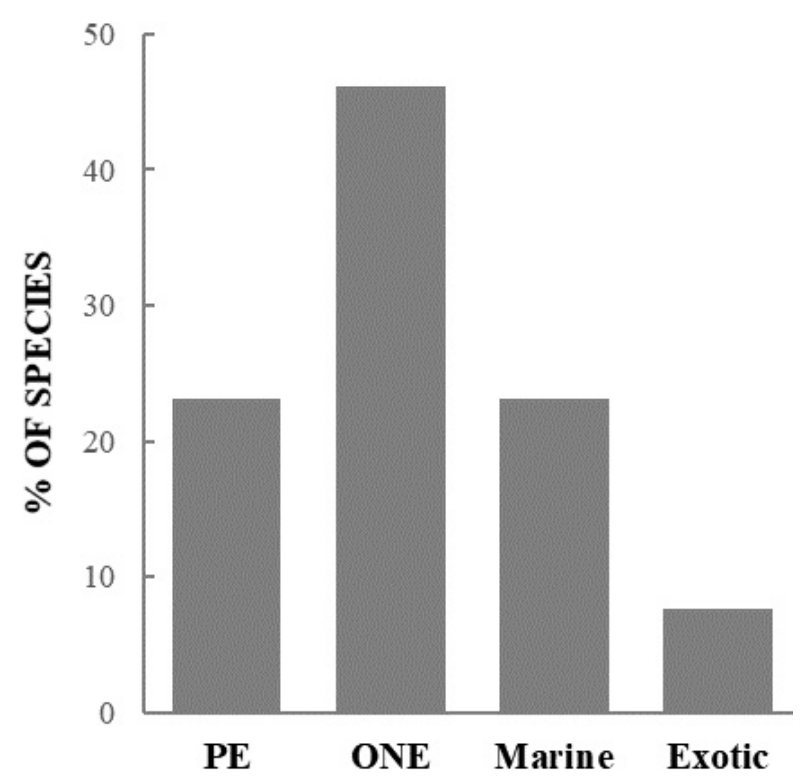

Figure 4. Number of species of each origin expressed as percentages of the total of species captured in the lower course of the Negro river drainage. Abbreviations: ONE, other Neotropical ecoregion; PE, native from Patagonia ecoregion.
Aug. 2016; F. Quezada leg.; MPCN-Ict 28. • 1 \%, 54 $\mathrm{mm}$; Site 4; 4043'15"S, 06336'02"W; 23 Aug. 2016; F. Guardiola Rivas leg.; MPCN-Ict 29. • 1 đ̃, 57 mm; Site 4; 4043'15"S, 063 $36^{\prime} 02^{\prime \prime} \mathrm{W}$; 20 Feb. 2017; M. Soricetti leg.; MPCN-Ict 30.

Identification. Body compressed. Premaxilla with one row of five multicuspidated teeth. Maxilla with one tooth. Pseudotympanum present. Lateral line interrupted with 5-8 perforated scales. Twenty-two to 29 ventral procurrent rays, more prominent in males. Pelvic fin with six branched rays. Caudal-fin rays, 14-17. Conspicuous subcircular dark spot on caudal peduncle present.

\section{Psalidodon pampa (Casciotta, Almirón \&}

Azpelicueta, 2005)

Figure 3D

Material examined. ARGENTINA • 1 q, $73 \mathrm{~mm}$; Site 2; 404' $48^{\prime \prime} \mathrm{S}, 063^{\circ} 23^{\prime} 08^{\prime \prime} \mathrm{W} ; 22$ Nov. 2016; M. Soricetti leg.; MPCN-Ict 4. • 1 \%, $47 \mathrm{~mm}$; Site 2; 4042'48"S, $063^{\circ} 23^{\prime} 08^{\prime \prime} \mathrm{W}$; 22 Nov. 2016; M. Soricetti leg.; MPCN-

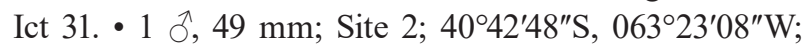
22 Nov. 2016; P. Solimano leg.; MPCN-Ict 32. • 1 q, 53

Table 2. List of species collected in the lower course of the Negro river drainage, Argentina. Abbreviations: ONE, other Neotropical ecoregion; PE, Patagonia ecoregion.

\begin{tabular}{|c|c|c|c|c|c|c|c|c|c|}
\hline \multirow{2}{*}{ Order/family/species } & \multirow{2}{*}{ Origin } & \multicolumn{7}{|c|}{ Sampling sites } & \multirow{2}{*}{ Collection code } \\
\hline & & 1 & 2 & 3 & 4 & 5 & 6 & 7 & \\
\hline \multicolumn{10}{|l|}{ Atheriniformes } \\
\hline \multicolumn{10}{|l|}{ Atherinopsidae } \\
\hline Odontesthes bonariensis (Valenciennes, 1835) & ONE & & & & $x$ & & $x$ & & MPCN-Ict 1, 14 to 18 \\
\hline Odontesthes hatcheri (Eigenmann, 1909) & $\mathrm{PE}$ & $x$ & & & $x$ & & & & MPCN-Ict 2, 19 to 24 \\
\hline \multicolumn{10}{|l|}{ Characiformes } \\
\hline \multicolumn{10}{|l|}{ Characidae } \\
\hline Cheirodon interruptus (Jenyns, 1842) & ONE & & $x$ & & $x$ & & & & MPCN-Ict 3, 25 to 30 \\
\hline Psalidodon pampa (Casciotta, Almirón \& Azpelicueta, 2005) & ONE & & $x$ & & $x$ & & & & MPCN-Ict 4, 31 to 42 \\
\hline \multicolumn{10}{|l|}{ Cypriniformes } \\
\hline \multicolumn{10}{|l|}{ Cyprinidae } \\
\hline Cyprinus carpio Linnaeus, 1758 & Exotic & $x$ & $x$ & & $x$ & & $x$ & $x$ & MPCN-Ict 5, 43 to 46 \\
\hline \multicolumn{10}{|l|}{ Cyprinodontiformes } \\
\hline \multicolumn{10}{|l|}{ Anablepidae } \\
\hline Jenynsia lineata (Jenyns, 1842) & ONE & $x$ & $x$ & $x$ & $x$ & & & & MPCN-Ict 6, 47 to 55 \\
\hline \multicolumn{10}{|l|}{ Poeciliidae } \\
\hline Cnesterodon decemmaculatus (Jenyns, 1842) & ONE & & $x$ & $x$ & & $x$ & & & MPCN-Ict 7, 56 to 65 \\
\hline \multicolumn{10}{|l|}{ Galaxiiformes } \\
\hline \multicolumn{10}{|l|}{ Galaxiidae } \\
\hline Galaxias maculatus (Jenyns, 1842) & $\mathrm{PE}$ & & $x$ & & & & & & MPCN-Ict 9, 66 \\
\hline \multicolumn{10}{|l|}{ Mugiliformes } \\
\hline \multicolumn{10}{|l|}{ Mugilidae } \\
\hline Mugil liza (Valenciennes, 1863) & Marine & & & & & & & $x$ & MPCN-Ict 8 \\
\hline \multicolumn{10}{|l|}{ Perciformes } \\
\hline \multicolumn{10}{|l|}{ Percichthyidae } \\
\hline Percichthys trucha (Cuvier \& Valenciennes, 1840) & $\mathrm{PE}$ & $x$ & & & $x$ & & & & MPCN-Ict 10,67 to 74 \\
\hline \multicolumn{10}{|l|}{ Pleuronectiformes } \\
\hline \multicolumn{10}{|l|}{ Paralichthyidae } \\
\hline Paralichthys brasiliensis (Ranzani, 1840) & Marine & & $x$ & & & & & $x$ & MPCN-Ict 11, 75, 76 \\
\hline \multicolumn{10}{|l|}{ Siluriformes } \\
\hline \multicolumn{10}{|l|}{ Ariidae } \\
\hline Genidens barbus (Lacepède, 1803) & Marine & & & & & & $x$ & & MPCN-Ict 13,82 to 85 \\
\hline \multicolumn{10}{|l|}{ Callichthyidae } \\
\hline Corydoras paleatus (Jenyns, 1842) & ONE & & $x$ & & $x$ & & & & MPCN-Ict 12,77 to 81 \\
\hline
\end{tabular}




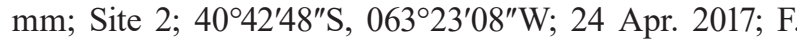
Guardiola Rivas leg.; MPCN-Ict 33. • 1 ふ, 62 mm; Site 2; 4042'48"S, 063²3'08"W; 24 Apr. 2017; M. Soricetti leg.; MPCN-Ict 34. • 1 9, 74 mm; Site 2; 4042'48"S, $063^{\circ} 23^{\prime} 08^{\prime \prime} \mathrm{W} ; 22$ Nov. 2016; M. Soricetti leg.; MPCN-Ict

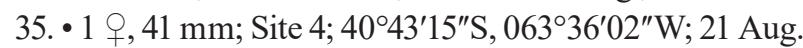
2016; F. Guardiola Rivas leg.; MPCN-Ict 36. • 1 ð, 45 mm; Site 4; 4043'15"S, 06336'02"W; 30 May 2017; M. Soricetti leg.; MPCN-Ict 37. • 1 §, 48 mm; Site 4; 4043'15"S, $063^{\circ} 36^{\prime} 02^{\prime \prime}$; 23 May 2017; P. Solimano leg.; MPCN-Ict

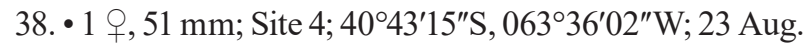
2016; M. Soricetti leg.; MPCN-Ict 39.・ 1 đ̄, 51 mm; Site 4; $40^{\circ} 43^{\prime} 15^{\prime \prime} \mathrm{S}, 063^{\circ} 36^{\prime} 02^{\prime \prime} \mathrm{W}$; 21 Aug. 2016; F. Guardiola Rivas leg.; MPCN-Ict 40. • 1 \%, 51 mm; Site 4; 4043'15"S, $063^{\circ} 36^{\prime} 02^{\prime \prime} \mathrm{W} ; 21$ Aug. 2016; M. Soricetti leg.; MPCN-

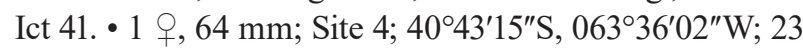
May. 2017; P. Solimano leg.; MPCN-Ict 42.

Identification. Body compressed. Dorsal and ventral profile of body slightly convex. Mouth terminal. Lower jaw slightly prognathous. Two rows of teeth on premaxilla; inner row with five tricuspidate or pentacuspidate teeth; single small and unicuspidate or tricuspidate tooth in the maxillary bone. Dentary with three or four large pentacuspidate teeth followed by three to four small teeth. Third infraorbital not contacting laterosensory canal of preopercle. Lateral line complete with 35-37 perforated scales. Four scales in contact with the supraoccipital bone. Anal fin with iii-v, 17-20 rays.

Order Cypriniformes

Family Cyprinidae

\section{Cyprinus carpio Linnaeus, 1758}

Figure 3E

Material examined. ARGENTINA• 1 \%, $418 \mathrm{~mm}$; Site 1; 40²6'18"S, 06340'54"W; 21 Jan. 2016; M. Soricetti leg.; MPCN-Ict 5. • 1 ग̃, 72 mm; Site 2; 4042'48"S; $063^{\circ} 23^{\prime} 08^{\prime \prime} \mathrm{W} ; 27$ Sep. 2016; M. Soricetti leg.; MPCN-

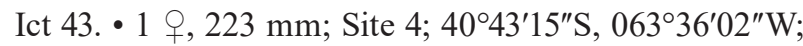
9 Apr. 2016; P. Solimano leg.; MPCN-Ict 44. • 1 ふૈ, 315 $\mathrm{mm}$; Site 6; 4048'10"S, 062 59'36"W; 24 Nov. 2016; B. Rodriguez leg.; MPCN-Ict 45. • 1 q, 584 mm; Site 7; $41^{\circ} 01^{\prime} 12^{\prime \prime} \mathrm{S}, 062^{\circ} 47^{\prime} 54^{\prime \prime} \mathrm{W} ; 25$ Oct. 2016; B. Rodriguez leg.; MPCN-Ict 46.

Identification. Robust body. Mouth edentulous, pharyngeal teeth present. Gill openings widely adhered to the isthmus. Scales large, 38 or 39 lateral-line scales. Dorsal fin long, with three or four unbranched anterior rays, the last of one bearing denticles. Caudal fin with rounded lobes. Greenish brown background color with golden and blue reflections.

Order Cyprinodontiformes

Family Anablepidae

Jenynsia lineata (Jenyns, 1842)

Figure 3F

Material examined. ARGENTINA• 1 q, $59 \mathrm{~mm}$; Site
1; 40²6'18"S, 06340'54"W; 21 Jan. 2016; M. Soricetti leg.; MPCN-Ict 6. • 1 ô, 23 mm; Site 1; 40²6'18"S, $063^{\circ}$ 40'54"W; 21 Jan. 2016; F. Guardiola Rivas leg.; MPCN-

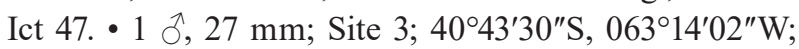
23 Apr. 2016; M. Soricetti leg.; MPCN-Ict 48. • 1 ,

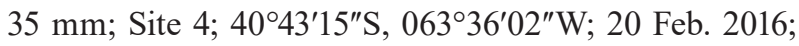
P. Solimano leg.; MPCN-Ict 49. • 1 q, $37 \mathrm{~mm}$; Site 2; $40^{\circ} 42^{\prime} 48^{\prime \prime} \mathrm{S}, 063^{\circ} 23^{\prime} 08^{\prime \prime} \mathrm{W}$; 13 Dec. 2016; M. Soricetti leg.; MPCN-Ict 50. • 1 \%, $43 \mathrm{~mm}$; Site 3; $40^{\circ} 4$ 3'30"S, 06314'02"W; 30 Apr. 2016; F. Guardiola Rivas leg.; MPCN-Ict 51. • 1 Oे, 49 mm; Site 4; 4043'15"S, $063^{\circ} 36^{\prime} 02^{\prime \prime} \mathrm{W} ; 23$ May. 2016; M. Soricetti leg.; MPCNIct 52. • 1 \%, $52 \mathrm{~mm}$; Site 2; 40 $42^{\prime} 48^{\prime \prime} \mathrm{S}, 063^{\circ} 23^{\prime} 08^{\prime \prime} \mathrm{W}$; 22 Nov. 2016; P. Solimano leg.; MPCN-Ict 53. • 1 ð, 55 mm; Site 1; 40²6'18"S, 06340'54"W; 18 Mar. 2016; F. Guardiola Rivas leg.; MPCN-Ict 54. • 1 \%, 58 mm; Site 1; 40²6'18"S, 06340'54"W; 23 May. 2017; M. Soricetti leg.; MPCN-Ict 55.

Identification. Small, body subcylindrical. A large central scale on the dorsal surface of the head. Predorsal series with 13 or 14 scales. Lateral line with 30-32 scales. A tubular gonopodium in the males formed by modified rays of the anal fin. Anal fin with 13 or 14 rays. Light gray background color with dash-shaped spots arranged in longitudinal lines, with four or five lines on the caudal peduncle.

Family Poeciliidae

\section{Cnesterodon decemmaculatus (Jenyns, 1842) Figure 3G}

Material examined. ARGENTINA 1 , $36 \mathrm{~mm}$; Site 2; 404' $48^{\prime \prime} \mathrm{S}, 063^{\circ} 23^{\prime} 08^{\prime \prime} \mathrm{W}$; 22 Nov. 2016; M. Soricetti leg.; MPCN-Ict 7. • 1 \%, 22 mm; Site 5; 4049'26"S, $063^{\circ} 09^{\prime} 51^{\prime \prime} \mathrm{W}$; 20 Feb. 2016; M. Soricetti leg.; MPCN-

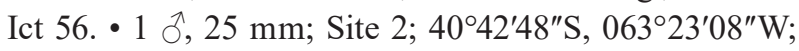
13 Dec. 2016; B. Rodriguez leg.; MPCN-Ict 57. • 1 +, $26 \mathrm{~mm}$; Site 5; 4049'26"S, 06309'51"W; 20 Feb. 2016; F. Guardiola Rivas leg.; MPCN-Ict 58. • 1 ô, 29 $\mathrm{mm}$; Site 3; 4043'30"S, 06314'02"W; 23 Apr. 2016; P. Solimano leg.; MPCN-Ict 59. • 1 q, 30 mm; Site 3; $40^{\circ} 43^{\prime} 30^{\prime \prime} \mathrm{S}, 063^{\circ} 14^{\prime} 02^{\prime \prime} \mathrm{W}$; 23 Apr. 2016; M. Soricetti leg.; MPCN-Ict 60. • 1 क, $31 \mathrm{~mm}$; Site 2; 4042'48"S, $063^{\circ} 23^{\prime} 08^{\prime \prime} \mathrm{W}$; 24 Apr. 2017; M. Soricetti leg.; MPCN-

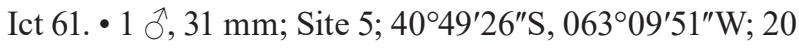
Feb. 2016; F. Guardiola Rivas leg.; MPCN-Ict 62. • 1 गै, $32 \mathrm{~mm}$; Site 3; 4043'30"S, 063¹4'02"W; 23 Apr. 2016; M. Soricetti leg.; MPCN-Ict 63. • 1 q, 33 mm; Site 5; 4049'26"S; 06309'51"W; 20 Feb. 2016; F. Guardiola Rivas leg.; MPCN-Ict 64. • 1 ô, 37 mm; Site 3; 4043'30"S, $063^{\circ} 14^{\prime} 02^{\prime \prime} \mathrm{W} ; 23$ Apr. 2016; P. Solimano leg.; MPCN-Ict 65.

Identification. Small, body subcylindrical. Snout acute. Lower jaw slightly longer than upper jaw. Longitudinal series with 31-33 scales. Anal-fin rays of males modified forming a gonopodium. Adult males gonopodium with a bony style long and slightly arched, covered by a 
membrane that ends in a distal filament. Grayish background color with dark bars, regularly spaced and restricted to the midline, along the sides of the body.

Order Galaxiiformes

Family Galaxiidae

\section{Galaxias maculatus (Jenyns, 1842)}

Figure $3 \mathrm{H}$

Material examined. ARGENTINA • 1 ๙, $57 \mathrm{~mm}$; Site

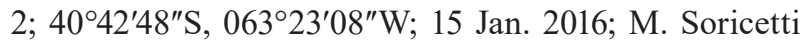
leg.; MPCN-Ict 9. • 1 क, $51 \mathrm{~mm}$; Site 2; 4042'48"S, $063^{\circ}$ 23'08"W; 24 Apr. 2017; P. Solimano leg.; MPCN-Ict 66.

Identification. Body elongated. Head short. Teeth almost equal in size, without differentiated canines. Maxilla reaches or slightly surpasses the anterior edge of the eye. The anal-fin origin is at the same level that the dorsal-fin origin, and when reclined, it does not reach the caudal fin. The pelvic-fin origin is closer to the base of the caudal fin than to the anterior end of the snout. Olive background, with irregular blackish spots, some of which are somewhat semilunar.

Order Mugiliformes

Family Mugilidae

\section{Mugil liza (Valenciennes, 1863)}

Figure 3I

Material examined. ARGENTINA • 1 , $501 \mathrm{~mm}$; Site 7; 4101'12"S, 06247'54"W; 26 Feb. 2017; S. Morawicki, P. Solimano leg.; MPCN-Ict 8.

Identification. Body elongated, fusiform, anteriorly depressed until the first dorsal-fin origin; caudal peduncle compressed. Head wide and depressed, interorbital space wide and convex. Mouth, wide and subterminal. Small labial teeth arranged in very close rows. Large ctenoid scales. Axillary scales of pectoral and pelvic fins less than a half the length of the respective fin. First dorsal fin equidistant between the snout tip and the origin of the caudal fin. Caudal fin finely scaled, almost to its distal edge. Dark brown color on back with flanks and belly silver; the scales of the back and flank with a more or less perceptible darker longitudinal groove.

Order Perciformes

Family Percichthyidae

\section{Percichthys trucha (Cuvier \& Valenciennes, 1840) Figure 3J}

Material examined. ARGENTINA • 1 o, $272 \mathrm{~mm}$; Site 1; 40²6'18"S, 06340'54"W; 21 Jan. 2016; M. Soricetti leg.; MPCN-Ict 10. • 1 O’, $61 \mathrm{~mm}$; Site 1; 40²6'18"S, 06340'54"W; 21 Jan. 2016; M. Soricetti, F. Guardiola Rivas leg.; MPCN-Ict 67. • 1 q, 66 mm; Site 1; 40²6'18"S, 06340'54"W; 21 Jan. 2016; M. Soricetti, F. Guardiola Rivas leg.; MPCN-Ict 68. • 1 +, 69 mm; Site 1; 40²6'18"S, 06340'54"W; 21 Jan. 2016; M. Soricetti, F. Guardiola Rivas leg.; MPCN-Ict 69. • 1 §, $71 \mathrm{~mm}$; Site 4; 4043'15"S, $063^{\circ} 36^{\prime} 02^{\prime \prime} \mathrm{W}$; 20 Feb. 2016; M. Soricetti, F. Guardiola Rivas leg.; MPCN-Ict 70.・1 9 , $78 \mathrm{~mm}$; Site 1; 40²6'18"S, 06340'54"W; 21 Jan. 2016; M. Soricetti, F. Guardiola Rivas leg.; MPCN-Ict 71. • 1 +, 95 mm; Site 4; 4043'15"S,

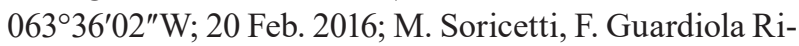
vas leg.; MPCN-Ict 72. • 1 ô, 102 mm; Site 1; 40²6'18"S, 06340'54"W; 21 Jan. 2016; M. Soricetti, F. Guardiola Rivas leg.; MPCN-Ict 73. • 1 \%, 142 mm; Site 4; 4043'15"S, $063^{\circ} 36^{\prime} 02^{\prime \prime} \mathrm{W}$; 20 Feb. 2016; M. Soricetti, F. Guardiola Rivas leg.; MPCN-Ict 74.

Identification. Body elongated and relatively compressed. Head length about three times of the body length. Mouth not very large, with the jaw included, which does not protrude above; maxilla relatively small, fitting 2.5 to 3 times into the head length, its posterior edge reaches the anterior edge of the eye. Wide interorbital space. Dorsal fin with 9-11 spines and 12 or 13 soft rays, the spiny and soft portions separated by a V-shaped notch. Small opercular spines; scapular process little robust. Anal fin with three spines and 9 or 10 soft rays. Its origin coincides with the end of the spinous portion of the dorsal fin. The color of live specimens is pale gold or lemon, up to golden olive, the belly is pale violet and the back may have olive shades to gray green or slate blue. All the scales have blue-black spots. Back of the head violaceous-brownish, and the operculum reddish. Dorsal fin light violet with brown spots and the caudal fin violet lemon with small dark specks.

Order Pleuronectiformes

Family Paralichthyidae

\section{Paralichthys brasiliensis (Ranzani, 1840)}

Figure 3K

Material examined. ARGENTINA • 1 o, $385 \mathrm{~mm}$; Site 7; 41 ${ }^{\circ} 01^{\prime} 12^{\prime \prime} \mathrm{S}, 062^{\circ} 47^{\prime} 54^{\prime \prime} \mathrm{W}$; 26 Feb. 2017. S. Morawicki, P. Solimano leg.; MPCN-Ict 11. • 1 q, 235 mm; Site 2; $40^{\circ} 42^{\prime} 48^{\prime \prime}$ S, $063^{\circ} 23^{\prime} 08^{\prime \prime} \mathrm{W}$; 4 Oct. 2017; M. Soricetti, S. Morawicki leg; MPCN- Ict 75. • 1 ふ, 406 mm; Site 7; $41^{\circ} 01^{\prime} 12^{\prime \prime} \mathrm{S}, 062^{\circ} 47^{\prime} 54^{\prime \prime} \mathrm{W} ; 26$ Feb. 2017. S. Morawicki, P. Solimano leg.; MPCN-Ict 76.

Identification. Body compressed. Cycloid scales on both sides of the body; 3-6 upper gill rakers and lower 13-18 in first branchial arch; 68-82 rays in dorsal fin; 50-61 rays in anal fin; 9-12 pectoral fin rays; 90-107 perforated scales on lateral line and 61-71 oblique rows of scales; small eyes (2.2-6\% SL) separated by flat, wide interorbital region (interorbital width $1.9-5 \% \mathrm{SL}$ ); short pectoral fins (pectoral fin on the ocular side $8.7-15.3 \%$ $\mathrm{SL})$; and narrow caudal peduncle (10.2-14.0\% SL).

Note. Paralichthys orbignyanus has been frequently confused with $P$. brasiliensis, the other species of the genus occurring in the South Atlantic. Paralichthys orbignyanus is distinguished from $P$. brasiliensis by the number of gill rakers in the first branchial arch (16-24 vs. $12-15$, respectively), the number of scales on the lateral line (90-107 vs. 81-88, respectively) and the oblique 
rows of scales (61-71 vs. 49-54, respectively), the shorter pectoral fins (average $11.5 \%$ vs. 13.1\% SL, respectively), the interorbital width (average 3.5\% vs. $2.7 \% \mathrm{SL}$, respectively) and the caudal peduncle width (average 11.6\% vs. $14.3 \%$ of the SL, respectively).

Order Siluriformes

Family Ariidae

\section{Genidens barbus (Lacepède, 1803)}

Figure 3L

Material examined. ARGENTINA • 1 ㅇ, $320 \mathrm{~mm}$; Site

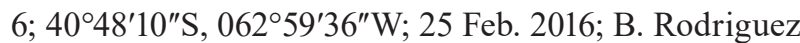
leg.; MPCN-Ict 13. • 1 9, 343 mm; Site 6; 4048'10"S, $062^{\circ} 59^{\prime} 36^{\prime \prime} \mathrm{W} ; 24$ Nov. 2016; B. Rodriguez leg.; MPCN-

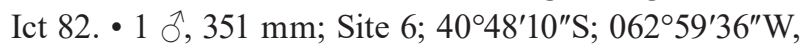
13 Dec. 2016; M. Soricetti leg.; MPCN-Ict 83.・ 1 ๆ, 364 $\mathrm{mm}$; Site 6; 4048'10"S; 062 ${ }^{\circ} 59^{\prime} 36^{\prime \prime} \mathrm{W}, 30$ Nov. 2017; M. Soricetti leg.; MPCN-Ict 84. • 1 +, 372 mm; Site 6; $40^{\circ} 48^{\prime} 10^{\prime \prime} \mathrm{S}, 062^{\circ} 59^{\prime} 36^{\prime \prime} \mathrm{W}$; 25 Feb. 2016; B. Rodriguez leg.; MPCN-Ict 85.

Identification. Body robust and elongated. The anterior frontal fontanelle reaches close to the base of the postoccipital process like a narrow groove. Upper jaw projected and lips thick. Maxillary barbels reaches the end of the operculum; the shortest mental and postmental barbels are inserted parallel to the edge of the jaw. Adipose fin, short. Pectoral-fin spine similar in length to dorsal-fin spine, with toothed internal edge. Caudal fin forked, with upper lobe narrower and longer than lower lobe. Anal fin emarginated. Uniform gray-black color, with purple reflections.

\section{Family Callichthyidae}

\section{Corydoras paleatus (Jenyns, 1842)}

Figure 3M

Material examined. ARGENTINA • 1 ふै, $75 \mathrm{~mm}$; Site 2; 4042'48"S; 063²3'08"W; 26 Nov. 2016; P. Solimano leg.; MPCN-Ict 12. • 1 ก̃, 49 mm; Site 2; 4042'48"S, $063^{\circ}$ 23'08"W; 26 Nov. 2016; F. Guardiola Rivas leg.; MPCN-

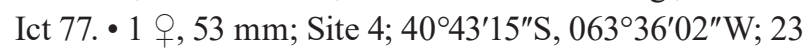
May. 2017; P. Solimano leg.; MPCN-Ict 78. 1 ठे, $61 \mathrm{~mm}$;

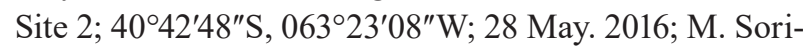

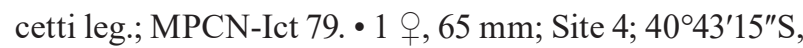
$063^{\circ} 36^{\prime} 02^{\prime \prime W}$; 23 May. 2017; M. Soricetti leg.; MPCN-Ict

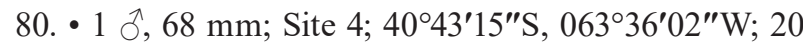
Feb. 2017; P. Solimano leg.; MPCN-Ict 81.

Identification. Body short and tall, flanks covered by two series of plates. Head compressed, triangular in dorsal view. Short maxillary barbels not reaching the gill opening. External mental barbels slightly longer than maxillary ones. Posterior margin of the pectoral spine serrated, perpendicularly oriented. Background color olive-brown with yellowish belly; three large black spots along the midline of flanks.

\section{Discussion}

Habitat destruction, pollution, overexploitation, climate change and invasive species have led to significant reductions in biological diversity (Dornelas et al. 2014). Invasive species are a prominent feature of many assemblages and have contributed to greater biotic homogenization. Communities are increasingly dominated by cosmopolitan species (Magurran 2016).

An example of this is the freshwater communities of Patagonia ecoregion, which are currently largely dominated by introduced fishes (Dyer 2000; Pascual et al. 2002). The richness of ichthyofauna in Patagonia ecoregion is low compared to other cold temperate regions of the southern hemisphere (Baigún and Ferriz 2003). Fifteen species have been introduced into Patagonia ecoregion from North America and Europe or have been translocated from other basins of Neotropical region, of which at least 11 have established self-sufficient populations (Pascual et al. 2007). Seven of the thirteen species sampled in the present study (54\%) do not belong to the Patagonia ecoregion. Baigún and Ferriz (2003) postulated that the species of other South America ecoregions present in the northern area of Patagonia ecoregion constitute $30 \%$ of the specific richness; however, at least in the lower course of the Negro river drainage this percentage is currently higher.

Menni et al. (1996) suggested that species such as Cnesterodon decemmaculatus and Jenynsia lineata had a wider distribution in the past. Baigún et al. (2002) stated that species such as Jenynsia lineata and Corydoras paleatus, due to their wide tolerance to variation in salinity and low temperatures, were able to disperse and colonize northern Patagonia ecoregion. Therefore, the presence and distribution of these species in Patagonia ecoregion can be explained by a combination of events and not by any single occurrence.

Ortubay et al. (1994) cited Cnesterodon decemmaculatus for the Curicó lagoon $\left(40^{\circ} 36^{\prime} 54^{\prime \prime} \mathrm{S}, 065^{\circ} 55^{\prime} 48^{\prime \prime} \mathrm{W}\right)$, as the southernmost record for the species. The present results imply an update of the distribution range of this species, extending it about $20 \mathrm{~km}$ to the south.

Pérez (2008) documented Psalidodon pampa in irrigation channels of Middle Valley of the Negro river drainage (approximately 39 $10^{\prime} 00^{\prime \prime} \mathrm{S}, \quad 066^{\circ} 9^{\prime} 00^{\prime \prime} \mathrm{W}$ ). The present report of $P$. pampa in the Lower Valley of the Negro river drainage constitutes the southernmost record for the species, extending its distribution range $180 \mathrm{~km}$ to the south. Casciotta et al. (2005) consider that $P$. pampa is the only species of the genus that inhabits rivers and streams south of the Bonaerensean drainages ecoregion. The references to Astyanax eigenmanniorum (Cope, 1894) (Baigún and Ferriz 2003; Pascual et al. 2007; Cussac et al. 2016) in the Patagonia ecoregion, probably correspond to $P$. pampa.

The fish fauna of Patagonia ecoregion will continue to be shaped by climate change and human disturbances (Cussac et al. 2004; Pascual et al. 2007), generating 
changes in the composition and relative abundance of the fish community (Pascual et al. 2007). In Patagonia ecoregion, at the present time, longitudinal systems are almost absent (Baigún and Ferriz 2003), which diminishes the dispersal capacity of species since there is no connection between the water freshwater bodies.

Artificial changes in the landscape, and introductions by live bait transport, aquarium hobby (Pérez and López Cazorla 2008), in addition to climate change, may facilitate the southward expansion of other invasive species.

\section{Acknowledgements}

We thank the Municipality of Guardia Mitre for the support provided during the development of this project, $\mathrm{Mr}$ Sergio Díaz for facilitating access to his field, and Bruno Rodríguez for his unconditional help in the sampling. Thanks also extend to the Facultad de Ciencias Naturales y Museo de La Plata, to Dr Jorge Casciotta for his collaboration in determination of the specimens, and to the Museo Patagónico de Ciencias Naturales "Juan Carlos Salgado" for catalog the samples in the collection. We also appreciate the financial support provided by the Universidad Nacional de Río Negro through PI 40-C683, 40-C-707 and 40-C-358. Finally, we appreciate the manuscript review of Audrey Urquhart BSc (Hons) Zoology. This manuscript benefited from the criticisms of two reviewers.

\section{Authors' Contributions}

All the authors conceived the research topic, collected data, and confirmed the taxonomic identity of the specimens. FQ and CG took the photographs. MS, PS, and AA wrote the first draft of the manuscript. SM and MS drew up the figures. FG and SM reviewed the final version of the manuscript.

\section{References}

Abell R, Thieme ML, Revenga C, Bryer M, Kottelat M, Bogutskaya N, Coad B, Mandrak N, Contreras Balderas S, Bussing W, Stiassny MLJ, Skelton P, Allen GR, Unmack P, Naseka A, Ng R, Sindorf N, Robertson J, Armijo E, Higgins VJ, Heibel TJ, Wikramanayake E, Olson D, López HL, Reis RE, Lundberg JG, Sabaj Pérez MH, Petry P (2008) Freshwater ecoregions of the world: a new map of biogeographic units for freshwater biodiversity conservation. BioScience 58 (5): 403-414. https://doi.org/10.1641/ B580507

Aigo J, Cussac V, Peris S, Ortubay S, Gómez S, López H, Gross M, Barriga J, Battini M (2008) Distribution of introduced and native fish in Patagonia (Argentina): patterns and changes in fish assemblages. Review in Fish Biology Fisheries 18: 387-408. https://doi. org/10.1007/s11160-007-9080-8

Albert JS, Reis R (2011) Historical biogeography of Neotropical freshwater fishes. University of California Press, Berkeley and London, 388 pp. https://doi.org/10.1525/california/97805202 68685.001 .0001

Almirón A, Azpelicueta M, Casciotta J, López Cazorla A (1997) Ichthyogeographic boundary between the Brazilian and Austral sub- regions in South America, Argentina. Biogeographica 73 (1): 23 30.

Almirón A, Casciotta J, Ciotek L, Giorgis P (2015) Guía de los peces del Parque Nacional Pre-Delta. Administración de Parques Nacionales, Ciudad Autónoma de Buenos Aires, 300 pp.

Alvear PA, Rechencq M, Macchi PJ, Alonso MF, Lippolt GE, Denegri MA, Navone G, Zattara EE, García Asorey MI, Vigliano PH (2007) Composición, distribución y relaciones tróficas de la ictiofauna del río Negro, Patagonia Argentina. Ecología Austral 17 (2): 231-246.

Aparicio FD, Difrieri HA (1958) La Argentina: suma de geografía. Ediciones Peuser. Buenos Aires, 457 pp.

Arratia G, Peñafort MB, Menu-Marque S (1983) Peces de la región sureste de los Andes y sus probables relaciones biogeográficas actuales. Deserta 7: 48-107.

Baigún C, Ferriz R (2003) Distribution patterns of native freshwater fishes in Patagonia, Argentina. Organisms, Diversity \& Evolution 3 (2): 151-159. https://doi.org/10.1078/1439-6092-00075

Baigún C, López GR, Dománico A, Ferriz RA, Sverlij S, Delfino Schenke R (2002) Presencia de Corydoras paleatus (Jenyns, 1842), una nueva especie brasílica en el norte de la Patagonia (río Limay) y consideraciones ecológicas relacionadas con su distribución. Ecología Austral 12 (1): 41-48.

Berasategui L (2002) Estadísticas climáticas del Valle de Viedma. 30 años. Información técnica 20 (2): 1-70.

Bruno MC, Casciotta JR, Almirón AE, Lizarralde MS (2013) Phylogeographic pattern of Jenynsia multidentata (Cyprinodontiformes: Anablepidae) in the southern boundary of the Brazilian Subregion, Argentina. Neotropical Ichthyology 11 (3): 477-486. https://doi.org/10.1590/S1679-62252013000300001

Casciotta J, Almirón A, Cione A, Azpelicueta M (1999) Brazilian freshwater fish assemblages from southern Pampean area, Argentina. Biogeographica 75 (2): 67-78.

Casciotta J, Almirón A, Azpelicueta M (2005) Astyanax pampa (Characiformes, Characidae), a new species from the southernmost boundary of the Brazilian subregion, Argentina. Revue Suisse de Zoologie 112 (2): 401-408. https://doi.org/10.5962/bhl. part. 80305

Cione AL, Barla MJ (1997) A new locality for the synbranchid eel Synbranchus marmoratus (Teleostei: Percomorpha) in southern Buenos Aires province, Argentina. Neotropica 43: 113-115.

Cussac V, Ortubay S, Iglesias G, Milano D, Lattuca M, Barriga J, Battini M, Gross M (2004) The distribution of South American galaxiid fishes: the role of biological traits and post glacial history. Journal of Biogeography 31 (1): 103-122. https://doi.org/10.1046/ j.0305-0270.2003.01000.x

Cussac VE, Fernández DA, Gómez SE, López HL (2009) Fishes of southern South America: a story driven by temperature. Fish Physiology and Biochemistry 35: 29-42. https://doi.org/10.1007/ s10695-008-9217-2

Cussac VE, Habit E, Ciancio J, Battini MA, Riva Rossi C, Barriga JP, Baigún C, Crichigno S (2016) Freshwater fishes of Patagonia: conservation and fisheries. Journal of Fish Biology 89 (1): 10681097. https://doi.org/10.1111/jfb.13008

Díaz de Astarloa JM, Munroe TA, Desoutter M (2006) Redescription and Holotype Clarification of Paralichthys orbignyanus (Valenciennes, 1839) (Pleuronectiformes: Paralichthyidae). Copeia 2006 (2): 235-243. https://doi. org/10.1643/0045-8511(2006)6[235:RAHCOP]2.0.CO;2

Dornelas M, Gotelli NJ, McGill B, Shimadzu H, Moyes F, Sievers C, Magurran AE (2014) Assemblage time series reveal biodiversity change but not systematic loss. Science, 344 (6181): 296-299. https://doi.org/10.1126/science.1248484

Dyer BS (2000) Systematic review and biogeography of the freshwater fishes of Chile. Estudios Oceanológicos 19: 77-98.

Géry J (1969) The fresh-water fishes of South America. In: Fittkau EJ, Illies J, Klinge H, Schwabe GH, Sioli H (Eds) Biogeography and 
ecology in South America. Dr W. Junk Publishers, Dordrecht, $828-848$.

Jackson DA (2002) Ecological effects of Micropterus Introductions: the dark side of Black Bass. In: Phillip DP, Ridgway MS (Eds) Black Bass: ecology, conservation, and management. American Fisheries Society Symposium, Bethesda, 221-232.

Koehn JD (2004) Carp (Cyprinus carpio) as a powerful invader in Australian waterways. Freshwater Biology 49 (7): 882-894. https:// doi.org/10.1111/j.1365-2427.2004.01232.x

Lodge DM (1993) Biological invasions: lessons for ecology. Trends in Ecology \& Evolution 8 (4): 133-137. https://doi.org/10.1016/016 9-5347(93)90025-K

Magurran AE (2016) How ecosystems change. Science 351 (6272): 448-449. https://doi.org/10.1126/science.aad6758

Malabarba LR, Malabarba MCSL (2008) Ictiofauna da Região Austral. Ciência \& Ambiente 35: 55-64.

Menni RC, Gómez SE, López Armengol FL (1996) Subtle relationships: freshwater fishes and water chemistry in southern South America. Hydrobiologia 328 (3): 173-197. https://doi.org/10.1007/ BF00017629

Miquelarena AM, Mantinian JE, López HL (2008) Peces de la Mesopotamia Argentina (Characiformes: Characidae: Cheirodontinae). INSUGEO, Serie Miscelánea 17 (1): 51-90.

Ortubay SG, Semenas LG, Ubeda CA, Quaggiotto AE, Viozzi GP (1994) Catálogo de peces dulceacuícolas de la Patagonia Argentina y sus parásitos metazoos. Dirección de. Pesca Subsecretaria de Recursos Naturales, Río Negro, 110 pp.

Pascual MA, Macchi P, Urbanski J, Marcos F, Riva Rossi C, Novara M, Dell'Arciprete P (2002) Evaluating potential effects of exotic freshwater fish from incomplete species presenceabsence data. Biological Invasions 4 (1-2): 101-113. https://doi. org/10.1023/A:1020513525528

Pascual MA, Cussac V, Dyer B, Soto D, Vigliano P, Ortubay S, Macchi $P$ (2007) Freshwater fishes of Patagonia in the 21st century after a hundred years of human settlement, species introductions, and environmental change. Aquatic Ecosystem Health \& Management 10 (2): 212-227. https://doi.org/10.1080/14634980701351361

Pérez CHF (2008) Fish, southernmost record of Astyanax pampa (Ostariophysi, Characiformes, Characidae). Check List 4 (4): 424-426. https://doi.org/10.15560/4.4.424

Pérez CHF, Lopez Cazorla AC (2008) Nuevos aportes al conocimiento de la ictiofauna del Rrío Negro, provincia de Río Negro, Argentina. Natura Neotropicalis 39 (1-2): 83-87. https://doi. org/10.14409/natura.v1i39.3869

Rahel FJ (2000) Homogenization of fish faunas across the United States. Science 288 (5467): 854-856. https://doi.org/10.1126/ science.288.5467.854

Reis RE, Albert JS, Di Dario F, Mincarone MM, Petry P, Rocha LA (2016) Fish biodiversity and conservation in South America. Journal of Fish Biology 89 (1): 12-47. https://doi.org/10.1111/jfb.13016

Ringuelet RA (1975) Zoogeografía y ecología de los peces de aguas continentales de la Argentina y consideraciones sobre las áreas ictiológicas de América del Sur. Ecosur 2 (3): 1-122.

Rosso JJ (2006) Peces pampeanos: guía y ecología. Literature of Latin America, Buenos Aires, 224 pp.

Vander Zanden MJ, Casselman JM, Rasmussen JB (1999) Stable isotope evidence for the food web consequences of species invasions in lakes. Nature 401 (6752): 464-467. https://doi. org $/ 10.1038 / 46762$

Wilcove DS, Rothstein D, Dubow J, Phillips A, Loso E (1998) Quantifying threats to imperiled species in the United States: assessing the relative importance of habit destruction, alien species, pollution, overexploitation, and disease. BioScience 48 (8): 607-615.

Zambrano L, Martínez-Meyer E, Menezes N, Peterson AT (2006) Invasive potential of Common Carp (Cyprinus carpio) and Nile Tilapia (Oreochromis niloticus) in American freshwater systems. Canadian Journal of Fisheries and Aquatic Sciences 63 (9): 19031910. https://doi.org/10.1139/F06-088 